\title{
Stress Driven Rearrangement Instability of Crystalline Films with Electromechanical Interaction
}

\author{
Peter Chung ${ }^{1}$, John Clayton ${ }^{2}$, Melanie M Cole ${ }^{3}$, Michael Grinfeld ${ }^{4}$, Pavel Grinfeld ${ }^{5}$, and \\ William Nothwang ${ }^{1}$ \\ ${ }^{1}$ AMSRD-ARL-CI-HC, US Army Research Laboratory, Aberdeen Proving Ground, MD, \\ 21005-5069 \\ ${ }^{2}$ AMSRD-ARL-WM-TD, US Army Research Laboratory, Aberdeen Proving Ground, \\ MD, 21005-5069 \\ ${ }^{3}$ AMSRD-ARL-WM-MA, US Army Research Laboratory, Aberdeen Proving Ground, \\ MD, 21005-5069 \\ ${ }^{4}$ AMSRD-ARL-WM-TD, US Army Research Laboratory, 4600 Deer Creek Loop, \\ Aberdeen Proving Ground, MD, 21005-5069 \\ ${ }^{5}$ Mathematics Department, Drexel University, Philadelphia, PA, 19104
}

\begin{abstract}
It was demonstrated, on general thermodynamic grounds, that, in non-hydrostatically stressed elastic systems, phase and grain interfaces undergo morphological destabilization due to different mechanisms of "mass rearrangement". Destabilization of free surfaces due to the combined action of mass rearrangement in the presence of electrostatic field has been well known since the end of the $19^{\text {th }}$ century. Currently, morphological instabilities of thin solid films with electro-mechanical interactions have found various applications in physics and engineering. In this paper, we investigate the combined effects of the stress driven rearrangement instabilities and the destabilization due to the electro-mechanical interactions. The paper presents relevant results of theoretical studies for ferroelectric thin films. Theoretical analysis involves highly nonlinear equations allowing analytical methods only for the initial stage of unstable growth. At present, we are unable to explore analytically the most important, deeply nonlinear regimes of growth. To avoid this difficulty, we developed numerical tools facilitating the process of solving and interpreting the results by means of visualization of developing morphologies.

\section{Introduction}

Recently, strong interest has focused on the development of tunable dielectric materials for frequency agile RF and microwave device applications [1]-[3]. Such electronic components include tunable filters, voltage controlled oscillators, varactors, parametric amplifiers, delay lines, and phase shifters [1]-[4]. Thin film $\mathrm{Ba}_{1-\mathrm{x}} \mathrm{Sr}_{\mathrm{x}} \mathrm{TiO}_{3}(\mathrm{BST})$ has come to be considered one of the forerunner material systems for the realization of such tunable components. In particular, it is the composition dependent Curie temperature $\left(\mathrm{T}_{\mathrm{C}}\right)$ and the nonlinear, field dependent, dielectric permittivity of $\mathrm{Ba}_{1-\mathrm{x}} \mathrm{Sr}_{\mathrm{x}} \mathrm{TiO}_{3}$ that makes it attractive for tunable devices at microwave frequency. In other words, BST is a material whose composition can be tuned such that under operating ambient, a nonhysteretic dielectric tunability can be produced through de bias.
\end{abstract}


Various sorts of defects can change dramatically the overall properties and macroscopic behavior of crystalline solids. Particular types of dominating defects vary from one large class of materials to another. It has been known for a long time that vacancies are especially important in ceramic materials [5]-[9]. Effects generated by nucleation, proliferation, and propagation of vacancies are important at all stages of production and exploitation of ceramic films. Mass transport through the bulk of solids is produced by the migration of vacancies, whereas near surfaces the migration of vacancies is accompanied by surface diffusion, vaporization-sublimation, etc. (see, for instance, [8]). The simplest and most robust features of the mechanism of surface diffusion have been studied in [10]. It is widely accepted that this mechanism dominates over migration of vacancies near traction-free surfaces. The situation can change dramatically at the nano-scale. At this level the very applicability of common thermodynamics seems questionable. Since at the nano-scale it is difficult to distinguish between the bulk and the surface part of the solid, it seems natural, at least, to withdraw existing macro- and microscale "obvious" pre-assumptions and to explore the mechanism of interaction between surface diffusion and vacancy migration.

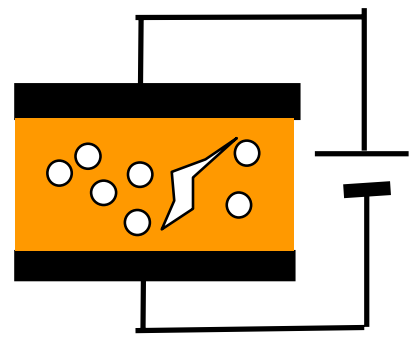

Figure 1. Ferroelectric solid film with charged vacancies.
Modeling of the behavior of ceramic thin films with vacancies in a framework of continuum mechanics and thermodynamics has been fulfilled in [11]-[13]. In particular, in [12], using one of the approaches of irreversible thermodynamics of solids, we explored the slow evolution of corrugations at the free boundary of a crystalline substance. The central issues of this study were the roles and the interaction of the three dissipation mechanisms: i) the surface diffusion of the species, ii) the bulk diffusion of vacancies, and iii) the flow of vacancies within the solid from vacuum. For the flux of vacancies from vacuum, we have postulated the constitutive equation, which can be easily justified on the basis of Onsager ideology (this means, basically, that the constitutive law for the flux should obey the linearity between the flux and corresponding driving force, and it has to provide the decay of the total accumulated energy). The model, formulated in [12], leads to deeply nonlinear problems. Therefore, only the linear stage of evolution of the solid films with vacancies has been analytically investigated in it. The much more interesting problems - more realistic from standpoint of applications - cannot be analyzed without numerical modeling.

Solid substances, analyzed in [11]-[13] were assumed to be electromagnetically neutral. For the active films applications these results should be improved by accounting for the electro-magneto-mechanical effects. Towards that goal, we plan to use thermodynamic extension of [11]-[13].

\section{Theoretical model}

In this note we analyze a capacitor with charged vacancies (Figure 1), and take into accout three dissipation mechanisms: i) dissipation from the flow of vacancies at the surface, ii) dissipation from bulk diffusion of vacancies, iii) dissipation from surface 
diffusion. In addition to the bulk energy $\varphi$, the Herring-like surface energy $\sigma$ is taken into account.

We consider a slightly corrugated solid film of thickness $H$ attached to a rigid substrate, see Figure 2. It is assumed that the vacancies can penetrate the bulk through the free interface $\Xi$ of the film only. We denote the time by $t$, the spatial coordinates by $x^{k}$ (the spatial - Latin - indices run 1,2,3, and summation over repeated indices is implied). Let $\chi\left(x^{k}, t\right)$ be the volume concentration of vacancies and $Q^{i}\left(x^{k}, t\right)$ is the vector of the volume flux of vacancies. We denote the surface coordinates of the free surfaces by $\xi^{\beta}$ (the surface - Greek - indices run 1,2); $c\left(\xi^{\alpha}, t\right)$ and $q^{\alpha}\left(\xi^{\beta}, t\right)$ are the normal velocity of the free surface and the surface flux of the species, respectively. Simple kinematics ([10], [8], [11]) leads to the following equations of mass conservation within the bulk:

$\frac{\partial \chi}{\partial t}=\frac{\partial Q^{i}}{\partial x^{i}}$

and at the free surface

$c(1-\chi)=Q^{i} n_{i}+\frac{\partial q^{\alpha}}{\partial \xi^{\alpha}}$,

where $n_{i}\left(\xi^{\alpha}, t\right)$ is a unit normal to the traction-free surface. The first term on the right hand side of (0.2) describes the migration of the free surface from the flow of vacancies through the surface, while the second describes the migration of the surface from the surface diffusion.

The bulk energy of the system is chosen in the simplest form:

$E=\int_{\Omega} d \Omega\left\{\phi\left(u_{i \mid j}, \chi\right)+V(x) \chi q\right\}+\int_{\Xi} d \Xi \sigma$,

where $u_{i \mid j} \equiv \partial u_{i}(x, t) / \partial x^{j}$ is a spatial displacement gradient; $V(x)$ is a potential of an external electric field, and $q$ is the electric charge per vacancy.

We postulate the following equations of mechanical equilibrium a) within bulk and b) at the traction-free interface:

a) $\left.\frac{\partial}{\partial x^{j}}\left(\frac{\partial \phi}{\partial u_{i \mid j}}\right)=0 \quad b\right) \frac{\partial \phi}{\partial u_{i \mid j}} n_{j}=0$.

The simplest "constitutive" equations of the volume and surface fluxes compatible with the Onsager principle of irreversible thermodynamics read:

$Q^{i}=D^{i j} \frac{\partial}{\partial x^{j}}\left(\phi_{\chi}+V q\right), q^{\alpha}=D^{\alpha \beta} \frac{\partial}{\partial \xi^{\alpha}} \frac{\phi+V q \chi-\sigma \kappa}{1-\chi}$,

where $D^{i j}$ and $D^{\alpha \beta}$ are the bulk and surface positive symmetric diffusion tensors, respectively; $\kappa$ is a doubled mean curvature of the surface. The simplest equation to describe the flow of vacancies, $Q^{i} n_{i}$, through the free surface compatible with the principle of energy dissipation reads:

$Q^{i} n_{i}=D_{I} \frac{\phi+V q-\sigma \kappa+(1-\chi) \phi_{\chi}}{1-\chi}$, 
where $D_{I}$ is a positive constant characterizing the resistance of the solid substance to penetrating of vacancies; $\phi_{\chi}=\partial \phi / \partial \chi$. For the sake of simplicity we assume that no vacancies flow through the film-substrate interface $\Sigma$ :

$Q^{i} n_{i}=0$.

The equations (0.1)-(0.7) form a closed, self-consistent, nonlinear system of the differential equations and boundary conditions for describing the slow evolution of deformable elastic solids with vacancies.

We denote by $\chi^{\circ}$ the equilibrium concentration of vacancies within the film when it has a plane free surface and is exposed to vacuum. Hence, according to (0.4), $\chi^{\circ}$ satisfies the equation $\varphi\left(\chi^{\circ}\right)+\left(1-\chi^{\circ}\right) \varphi_{\chi}\left(\chi^{\circ}\right)=0$.

Let us assume now that the free surface $\Xi$ is slightly corrugated. The evolution of this corrugation can be explored by linearizing the equations $(0.1)-(0.7)$ in the vicinity of the uniform configuration with further consideration of the solutions of the form $\sim \exp \left(\eta t+i k_{\alpha} \xi^{\alpha}\right)$; here $\xi^{\alpha}$ are the Cartesian coordinates on the equilibrium plane surface, $k_{\alpha}$ is the in-plane wave-number of the corrugation, and $r$ is the growth- decay rate of the corrugations.

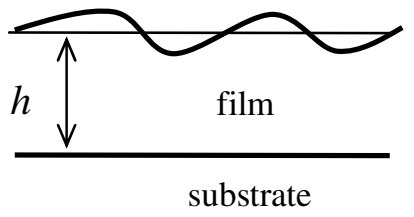

Figure 2. Film-substrate system

In the following, we assume that the film is isotropic and contains a small concentration of vacancies $\chi^{\circ} \ll \chi^{\circ}$. Hence, the diffusion tensors take on the form: $D_{b}^{i j}=D_{b} \delta^{i j}, D^{\alpha \beta}=D_{s} \delta^{\alpha \beta}$, where $D_{b}$ and $D_{s}$ are positive constants whereas $\delta^{i j}$ and $\delta^{\alpha \beta}$ are the spatial and surface Kronekker symbols. In the absence of stresses, a routine computation results in the following dispersion equation:

$$
\left[D_{b} k \tanh \left(k h \sqrt{1+\frac{\eta}{k^{2} N D_{b}}}\right)+D_{l}\right]\left(\eta+D_{s} \sigma k^{4}\right)+D_{b} D_{I} \sigma k^{3}=0
$$

where $N=\partial^{2} \varphi\left(\chi^{\circ}\right) / \partial \chi^{2}$. When $\eta /\left(k N D_{b}\right)<<1$, the equation (0.8) reads:

$$
\eta=-\sigma k^{3} \frac{D_{s} D_{b} k^{2} \tanh (k h)+D_{l} D_{s} k+D_{b} D_{I}}{D_{b} k \tanh (k h)+D_{l}} \text {. }
$$

According to (0.9) the three dissipative mechanisms are not additive.

Assume that the deformable, isotropic, and sufficiently thick film is subjected to the lateral stress $T$. In the absence of the external electric field, the master system leads to the following dispersion equation: 


$$
\begin{aligned}
& \left(D_{b} k \sqrt{1+\frac{\eta}{k^{2} N D_{b}}}+D_{i}\right) \eta+ \\
& {\left[\sqrt{1+\frac{\eta}{k^{2} N D_{b}}}\left(D_{k} D_{i}+D_{b} D_{s}\right) k^{2}+D_{i} D_{s} k^{3}\right] \times\left[\sigma k-(1-v) \frac{T^{2}}{\mu}\right]=0,}
\end{aligned}
$$

where $v$ is the Poisson ratio, $T$ is the uniaxial stress.

When $\eta /\left(k N D_{b}\right)<<1$, the equation (0.10) implies the relationship:

$$
\eta=-k^{2} \frac{D_{k} D_{i}+D_{b} D_{s}+D_{i} D_{s} k}{D_{b} k+D_{i}}\left[\sigma k-(1-v) \frac{T^{2}}{\mu}\right] \text {. }
$$

According to (0.11), stresses within the solid can destabilize flat interfaces when rearrangement of mass is allowed. The dispersion equation (0.11) is compatible with the static stability criteria of [14], [17] though it differs from the criterion of [18] exploring the same mechanism of rearrangement in pre-stressed crystal.

When the electric field does not vanish within the ferroelectric crystal, the dispersion equations appear to be too cumbersome for presenting in this short note. It becomes much more transparent in the case of a high dielectric constant. In this case, the crystalline film behaves like a metal. Assume, that i) the rearrangement is controlled by the evaporation/condensation mechanism with the dissipation constant $K$, and ii) the surface energy is proportional to the actual area of the traction-free surface. Then, the dispersion relation can be presented as:

$$
\eta=K\left(4 \pi \tau^{2} k-\sigma k^{2}+\frac{1-v}{\mu} k T^{2}\right)\left[1+(1-v) \frac{\sigma k}{2 \mu}\right]^{-1},
$$

where $\tau$ is the conductor's surface charge density. According to (0.12), there are two destabilizing mechanisms influencing the morphology of the film: i) the Rayleigh-TonksFrenkel' instability due to the electrostatic field [19]-[20] and ii) the instability of the "stressed crystal - melt".

\section{Conclusions}

Using one of the approaches of irreversible thermodynamics of solids we formulated a general master system allowing us to explore the slow evolution of charged vacances in a crystalline substance. The central issues of this study are the roles and the interaction of the three dissipation mechanisms: i) the surface diffusion of the species, ii) the bulk diffusion of vacancies, and iii) the flow of vacancies within the solid from vacuum. For the fluxes of vacancies we postulated the constitutive equations (0.5), (0.6), which can be justified on the basis of the Onsager principle of irreversible thermodynamics. With the help of the master system of slow evolution (0.1)-(0.7). The master system and its implications (0.9)-(0.12) allow one to distinguish between those corrugations for which the Mullins' $k^{4}$-law gives acceptable approximation and those for which it does not. The above equations clearly show that the three above mentioned dissipative mechanism cannot be described as the linear superposition even for small disturbances (as they are treated usually in the existing literature). Our dispersion equations differ considerably from the similar equation of [18]. At last, it was demonstrate that in the presence of an 
electrostatic field there appear two destabilizing mechanism opposed by surface tension: i) the instability "stressed crystal - melt" and ii) the Rayleigh-Tonks-Frenkel' instability due to the electrostatic field [19]-[20].

\section{References}

[1] M. W. Cole, R. G. Geyer, C. Hubbard, E. Ngo, M. Ervin, M. Wood, J. Appl. Phys. 92, 475 (2002) .

[2] B. H. Park, E. J. Peterson, Q. X. Jia, J. Lee, X. Zeng, W. Si, and X. X. Xi, Appl. Phys. Lett. 78, 533 (2001).

[3] M. W. Cole, W. D. Nothwang, C. Hubbard, E. NgO, and M. Ervin, J. Appl. Phys. 93, 9218 (2003).

[4] W. Chang, J. S. Horwitz, A. C. Carter, J. M. Pond, S. W. Kirchoefer, C. M. Gilmore, and D. B. Chrisey, Appl. Phys. Lett. 74, 1033 (1999).

[5] C. J. Herring, Appl. Phys., 21, 437 (1950).

[6] I. M. Lifshitz, Soviet Physics JETP 17, 909 (1963).

[7] C. E. Flynn, Point Defects and Diffusion (Clarendon Press, 1972).

[8] J. W. Christian, The Theory of Transformations in Metals and Alloys (Pergamon Press, 1975).

[9] Ya. E. Geguzin, Physics of Sintering (Nauka, 1984).

[10] W.W. Mullins, J. Appl. Phys. 28, 333 (1957).

[11] M. A. Grinfeld, P. Hazzledine, Phil. Mag. Lett. 74, 17 (1996).

[12] M.A. Grinfeld, P Hazzledine, Europhys. Lett. 37, 409 (1997).

[13] V. Berdichevsky, P. Hazzledine, B. Shoykhet, Int. J. Engng Sci. 35, 103 (1997).

[14] P. M. Grinfeld, M. A. Grinfeld, Ferroelectrics (2006) (in press).

[15] M. A. Grinfeld, Thermodynamic Methods in the Theory of Heterogeneous Systems (Longman, 1991).

[16] M. A. Grinfeld, Scanning Microscopy 8, 869 (1994).

[17] Ph. Nozières, "Shape and Growth of Crystals", Solids Far From Equilibrium, ed. C. Godrèche, Cambridge: (Camb. Univ. Press, 1991) pp. 1-153.

[18] P. Vasudev, , R. J. Asaro, and W.A . Tiller, Acta Metall. 23, 341 (1975).

[19] L. D. Landau, and E. M. Lifshitz, Electrodynamics of Continuous Media (Pergamon Press, 1984).

[20] L. Tonks, Phys. Rev. 48, 562 (1935). 